\title{
Variaciones estacionales de parámetros reproductivos, calidad espermática y enzimas del plasma seminal en carneros Texel
}

\author{
Ojeda, P.M.'; Manes, J.'; Aller, J.F. ${ }^{3}$ \\ 'EEA-INTA Catamarca, Argentina. ${ }^{2}$ EEA-INTA Marcos Juárez, Córdoba, \\ Argentina. ${ }^{3}$ EEA-INTA Balcarce, Buenos Aires, Argentina. \\ E-mail: alleratucha.juan@inta.gob.ar
}

\begin{abstract}
Resumen
Ojeda, P.M.; Manes, J.; Aller, J.F.: Variaciones estacionales de parámetros reproductivos, calidad espermática y enzimas del plasma seminal en carneros Texel. Rev. Vet. 32: 2, 138-145, 2021. El objetivo del presente trabajo fue determinar el efecto de la estación del año (fotoperíodo) sobre la circunferencia escrotal (CE), peso testicular (PT), volumen testicular (VT), capacidad de servicio (CS), concentración plasmática de testosterona, características cuali-cuantitativas del semen fresco y enzimas del plasma seminal de carneros de raza Texel. El estudio se realizó en la pampa húmeda de Argentina ( $\left.37^{\circ} 45^{\prime} \mathrm{S}, 58^{\circ} 18^{\prime} \mathrm{O}, 130 \mathrm{msnm}\right)$. Se utilizaron seis carneros jóvenes ( 2 años de edad) y las determinaciones realizadas, peso vivo, CE, PT [PT=0,5533 $\left.\times(\mathrm{L}) \times(\mathrm{A})^{2}\right]$, VT [VT=4/3 $\left.\times(\pi) \times(\mathrm{L} / 2) \times(\mathrm{A} / 2)^{2}\right]$ (siendo $\mathrm{L}=$ longitud $\mathrm{y}$ $\mathrm{A}=$ ancho del testículo) y testosterona plasmática se realizaron una vez por mes. La prueba de CS se realizó dos veces por estación y la evaluación del semen se efectuó dos veces por semana durante el mes central de cada estación. Las variables fueron analizadas mediante modelos lineales mixtos para medidas repetidas en el tiempo ( $R$ Core Team). En verano (Marzo) se observaron los mayores valores (media $\pm \mathrm{EE} ; \mathbf{p}<0,05)$ de $\mathrm{CE}(33,8 \pm 0,6 \mathrm{~cm})$, PT $(467,5 \pm 15,2$ g) y VT $\left(442,3 \pm 8,5 \mathrm{~cm}^{3}\right)$. El número promedio de montas totales fue mayor $(\mathrm{p}<0,05)$ en invierno (21) respecto de la primavera (9). La mayor concentración plasmática de testosterona $(\mathbf{p}<0,05)$ fue determinada en verano (Enero $=13,2 \pm 2,9 \mathrm{ng} / \mathrm{ml}$ ) y la menor en invierno (Septiembre $=0,13 \pm 0,1 \mathrm{ng} / \mathrm{ml})$. Eyaculados ( $\mathrm{n}=192)$ fueron evaluados y los mejores parámetros de calidad seminal fueron determinados en otoño e invierno. Los patrones anuales de las enzimas alanina-amino-transferasa y lactato-deshidrogenasa mostraron una significativa variación estacional $(\mathrm{p}<0,05)$, con la más alta actividad durante la estación reproductiva. Por otro lado, las concentraciones de aspartato-amino-transferasa, gama-glutamil-transpeptidasa y fosfatasa alcalina fueron más bajas en verano. Los resultados sugieren que los carneros Texel presentan un mayor potencial reproductivo en la estación de otoño (fotoperíodo decreciente) en esta latitud.
\end{abstract}

Palabras clave: carnero, capacidad de servicio, testosterona, calidad seminal, enzimas.

\begin{abstract}
Ojeda, P.M.; Manes, J.; Aller, J.F.: Seasonal variations of reproductive parameters, sperm quality and seminal plasma enzymes in Texel rams. Rev. Vet. 32: 2, 138-145, 2021. The objective of the present work was to determine the effect of season of the year (photoperiod) on scrotal circumference (SC), testicular weight (TW), testicular volume (TV), serving capacity test (SCT), plasma testosterone concentration, semen quality characteristics and seminal plasma enzymes in Texel rams. This study was carried out at the humid pampas of Argentina ( $37^{\circ} 45^{\prime} \mathrm{S}, 58^{\circ} 18^{\prime} \mathrm{W}$; $130 \mathrm{~m}$ above sea level). Six Texel young (2 years old) rams were used. Body weight, SC, TW [TW=0.5533 $\left.\times(\mathrm{L}) \times(\mathrm{W})^{2}\right]$, TV[TV=4/3 $\times(\pi) \times(\mathrm{L} / 2) \times$ $\left.(\mathrm{W} / 2)^{2}\right]$ (being $\mathrm{L}=$ long $\mathrm{y} \mathrm{W}=$ width of the testicle) and plasma testosterone concentrations were determined once a month and the SCT was performed twice per season of the year. Statistical analyses were carried out through linear mixed models for repeated measures ( $R$ Core Team). Evaluation of fresh semen was made twice weekly by four consecutive weeks during each season, starting from the 5th to 8th week (middle) of each season. The higher values (mean $\pm \mathrm{SE} ; \mathrm{p}<0.05)$ of the SC $(33.8 \pm 0.6 \mathrm{~cm})$, TW $(467.5 \pm 15.2 \mathrm{~g})$ and TV $\left(442.3 \pm 8.5 \mathrm{~cm}^{3}\right)$ were observed in the summer (March). The highest mean number $(p<0.05$ ) of total mounts was determined in the winter (21) compared to spring (9). Plasma testosterone concentration was decreasing from summer (January $=13.2 \pm 2.9 \mathrm{ng} / \mathrm{ml}$ ) to winter (September $=0.13 \pm 0.1 \mathrm{ng} /$ $\mathrm{ml})$. Ejaculates $(\mathrm{n}=192)$ were evaluated and the best-quality semen parameters were determined in autumm and winter. The yearly patterns of alanine-amino-transferase and lactate-dehydrogenase showed a significant seasonal variation $(\mathbf{p}<0.05)$ with a higher activity
\end{abstract}


during breeding season. On the other hand, concentrations of aspartate-amino-transferase, gama-glutamyl-transpeptidase and alkaline-phosphatase were lower $(\mathrm{p}<0.05)$ in summer. In conclusion, results suggest that Texel rams show the highest potential reproductive in the autumm season (decreasing photoperiod) in this latitude.

Key words: ram, Texel, serving capacity, testosterone, semen quality, enzymes.

\section{INTRODUCCIÓN}

La raza Texel fue creada hacia fines del siglo XIX en la isla Texel, al norte de Holanda, por medio de cruzamientos entre la raza Old Texel con Lincoln y Leicester Longwool e introducida en la Provincia de Buenos Aires desde Uruguay, en 1977.

El ovino es una especie con actividad reproductiva estacional (fotoperíodo decreciente) que se manifiesta en el otoño en latitudes lejanas al Ecuador. Por otro lado, las razas adaptadas a vivir en latitudes bajas, carecen de un "anestro estacional verdadero". En este contexto, se observó que la estación del año y/o el fotoperíodo decreciente afectan los parámetros reproductivos en razas como Texel, Suffolk, Karakul y Dorset ${ }^{12}$.

Durante el trayecto epididimario y la eyaculación, los espermatozoides entran en contacto con secreciones de las glándulas anexas (plasma seminal) donde adquieren motilidad progresiva y capacidad fertilizante ${ }^{26}$.

El plasma seminal presenta actividad antimicrobiana, neutraliza metabolitos espermáticos e interviene en la capacitación espermática ${ }^{27}$. En su composición se encuentran proteínas, enzimas, hormonas, iones, entre otras. Algunos autores han relacionado la actividad enzimática del plasma seminal con las características de calidad espermática del eyaculado ${ }^{15}$.

La información existente sobre la actividad reproductiva anual de los carneros de la raza Texel, es limitada y en ocasiones difícil de interpretar, debido a las diversas metodologías que se han utilizado para su estudio. La hipótesis de trabajo fue que las características reproductivas de los carneros Texel varían según la estación del año, siendo el otoño (fotoperíodo decreciente) la estación con mejor aptitud reproductiva en la latitud estudiada.

Por lo tanto, el objetivo del presente estudio fue determinar el efecto de la estación del año sobre los siguientes parámetros reproductivos: circunferencia escrotal, peso y volumen testicular, capacidad de servicio, concentración plasmática de testosterona, características cuali-cuantitativas del semen fresco y concentraciones enzimáticas en el plasma seminal.

\section{MATERIAL Y MÉTODOS}

Este trabajo se realizó en la EEA Balcarce del Instituto Nacional de Tecnología Agropecuaria (INTA), Provincia de Buenos Aires, Argentina (37\%45' latitud sur, $58^{\circ} 18^{\prime}$ longitud oeste, $130 \mathrm{msnm}$ ), desde el 21 de marzo de 2017 al 20 de marzo de 2018. Las estaciones del año consideradas fueron otoño (21/03-20/06), in- vierno (21/06-22/09), primavera (21/09-20/12) y verano $(21 / 12-20 / 03)$.

Se utilizaron seis carneros Texel de dos años de edad (dos dientes permanentes) manejados en un sistema de pastoreo rotativo de avena $(08.00 \mathrm{a} 15.00 \mathrm{~h})$ y suplementación a corral (15.00 a $07.00 \mathrm{~h}$ del día siguiente) con disponibilidad de agua ad libitum, seronegativos a Brucella ovis y Brucella melitensis.

Las variables físicas, peso vivo (PV) y circunferencia escrotal (CE) fueron determinadas una vez por mes. La CE fue medida con una cinta métrica metálica y cada testículo fue medido con un calibre electrónico, siendo la longitud el sentido más largo de la gónada, excluyendo cabeza y cola de epidídimo, y el ancho, la región ecuatorial de cada testículo.

Posteriormente se empleó la fórmula de peso testicular $\left[\mathrm{PT}=0,5533 \times(\mathrm{L}) \times(\mathrm{A})^{2}\right]$ y volumen testicular [VT=4/3 $\times(\pi) \times(L / 2) \times(A / 2)^{2}$ ], siendo $L=$ longitud $y$ $\mathrm{A}=$ ancho del testículo ${ }^{3} \mathrm{y}$ los resultados finales se expresan para ambos testículos.

La prueba de capacidad de servicio (CS) se realizó dos veces por estación del año, en forma individual a corral con tres ovejas ovariectomizadas sujetas en bretes de salto durante 20 minutos y se registró el número de montas totales y cópulas completas ${ }^{11}$

Se realizó extracción de sangre una vez por mes de la vena yugular, se centrifugó a $600 \mathrm{~g} / 10 \mathrm{~min}$ y el plasma fue separado y almacenado a $-20^{\circ} \mathrm{C}$ hasta la determinación de la concentración de testosterona por medio de radioinmunoanálisis (RIA, kit comercial IM1119, Beckman Coulter Inc.USA).

Todas las muestras se procesaron con un coeficiente de variación intra-ensayo menor al $9 \%$ para concentraciones comprendidas entre 0,09 y $22,0 \mathrm{ng} / \mathrm{ml}$ y una sensibilidad estimada de $0,10 \mathrm{ng} / \mathrm{ml}$.

El semen fue colectado con vagina artificial dos veces por semana durante las cuatro semanas centrales (quinta a octava semana) de cada estación y colocado en Baño María a $32^{\circ} \mathrm{C}$ para su evaluación. Las variables seminales evaluadas en semen fresco fueron: volumen, $\mathrm{pH}$, motilidad de masa microscópica (0 a 5), concentración espermática (millones de espermatozoides/ $\mathrm{ml}$ ), motilidad individual progresiva (\%), vigor (0 a 5), relación vivos/muertos (\%) y morfología espermática (\% de anormalidades).

Luego de la evaluación, un total de 48 eyaculados (12 por estación) se procesaron para la obtención del plasma seminal según la técnica de doble centrifugación ${ }^{2}$. El plasma seminal se almacenó a $-80^{\circ} \mathrm{C}$, hasta el momento de determinar las concentraciones de glucosa y de las enzimas aspartato-aminotransferasa 
(AST), alanina-aminotransferasa (ALT), gamma-glutamil-transpeptidasa (GGT), fosfatasa alcalina (ALP) y lactato-deshidrogenasa (LDH).

Los procedimientos sobre los animales se realizaron cumpliendo con las normas requeridas por el Comité Institucional para el Cuidado y Uso de Animales de Experimentación (CICUAE, Protocolo $\mathrm{N}^{\circ} 025$ ) del INTA.

Para el análisis estadístico de las variables se ajustaron modelos lineales mixtos para medidas repetidas en el tiempo. Se consideraron los meses/estaciones del año como efecto fijo y el carnero, como efecto aleatorio y en algunos casos se incorporó una estructura autoregresiva entre los errores del mismo carnero dentro de una misma estación.

La heterogeneidad de varianza se modeló en las variables donde fue necesario, para seleccionar el modelo adecuado, a su vez se realizaron gráficos de diagnóstico de los residuales y pruebas de cociente de máxima verosimilitud restringida, para comparar modelos en cada variable. Se realizaron pruebas de Tukey para determinar diferencias entre medias.

Se trabajó con un nivel de significación del $5 \%$ para considerar efectos significativos. Los análisis se efectuaron empleando el software R mediante la interfaz
RStudio (Versión 0.99.903) ${ }^{22}$, los gráficos se realizaron con el paquete lattice y los ajustes de modelos mixtos se realizaron con el paquete nlme.

\section{RESULTADOS}

El PV promedio ( \pm EE) anual de los animales fue de $60,4( \pm 0,8) \mathrm{kg}$, observándose el mayor valor $(\mathrm{p}<0,05)$ en el verano $(70,6 \pm 2,3 \mathrm{~kg})$.

Los promedios $( \pm \mathrm{EE})$ anuales de la $\mathrm{CE}$, el PT y el VT fueron $30,4( \pm 0,2) \mathrm{cm}, 331,1( \pm 7,9) \mathrm{g}$ y $313,4( \pm 7,5)$ $\mathrm{cm}^{3}$, respectivamente. En las estaciones de verano y otoño se observaron los mayores valores $(p<0,05)$ en los tres parámetros evaluados (Figura 1).

No se registraron diferencias $(\mathrm{p}>0,05)$ en el número promedio de cópulas completas entre estaciones (Figura 2). La eficiencia de servicio (cópulas completas/ montas totales $\mathrm{x} 100)$ fue menor $(p<0,05)$ en las estaciones de otoño e invierno ( 32 y $25 \%$ respectivamente) respecto de la primavera y el verano $(65$ y $55 \%$ respectivamente) (Figura 2).

Las concentraciones de testosterona plasmática fueron más bajas $(p<0,05)$ en otoño e invierno, hasta alcanzar un máximo valor $(p<0,05)$ al comienzo del ve-

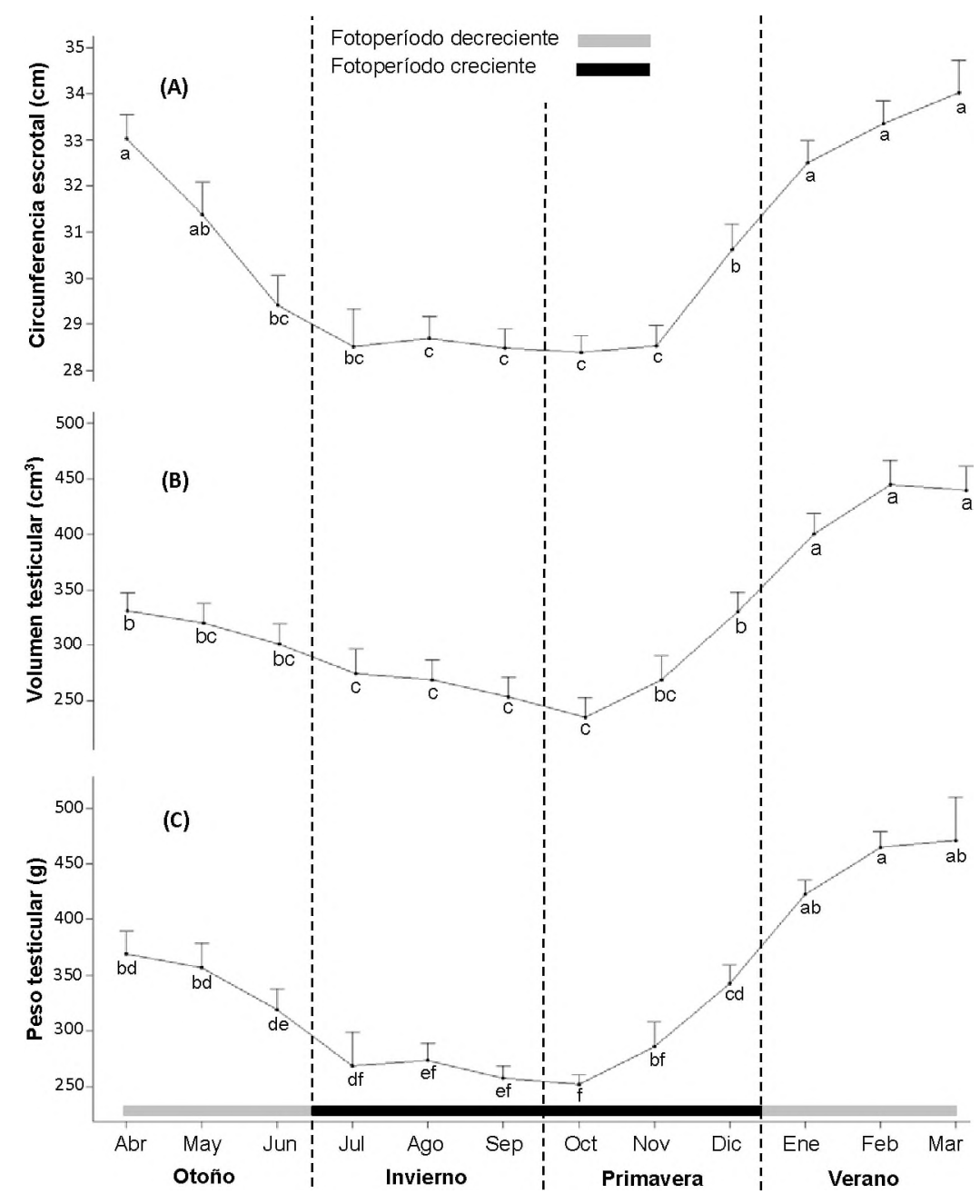

Figura 1. Variación mensual (promedio $\pm \mathrm{EE}$ ) de la circunferencia escrotal (A), volumen testicular (B) y peso testicular (C) de ambos testículos en carneros Texel. Letras diferentes indican diferencias significativas $(p<0,05)$. 
rano $(13,2 \mathrm{ng} / \mathrm{ml})$, para posteriormente disminuir hacia el final de la estación (Figura 3).

Los 192 eyaculados colectados de los carneros Texel fueron evaluados y los resultados de calidad espermática se presentan en la Tabla 1. Los promedios para las variables VOL, TESPZ, MIP y porcentaje de EV disminuyeron desde el máximo valor $(p<0,05)$ en el otoño hasta alcanzar el mínimo en la primavera. No se observaron diferencias $(p>0,05)$ entre estaciones en la concentración espermática (CONC), el pH y el Vigor.

Tabla 2: el porcentaje de espermatozoides normales $(E N)$ fue similar $(p>0,05)$ entre estaciones. El porcentaje de espermatozoides con anormalidades a nivel de la cabeza (EACA) se incrementó desde el otoño/invierno hasta alcanzar el máximo $(\mathrm{p}<0,05)$ en el verano. En el otoño se observaron los mayores $(p<0,05)$ porcentajes de espermatozoides con anormalidades a nivel de la pieza intermedia (EAPI) y cola (EAC).

La glucosa presentó la menor concentración en el otoño ( $p<0,05)$ respecto del resto de las estaciones. Las enzimas AST, GGT y ALP presentaron las menores concentraciones en el verano $(p<0,05)$. En contraste, la enzima ALT presentó las mayores concentraciones en el verano y el otoño $(p<0,05)$; a su vez, la enzima $L D H$ presentó una mayor concentración en el otoño $(p<0,05)$, como indica la Tabla 3 .

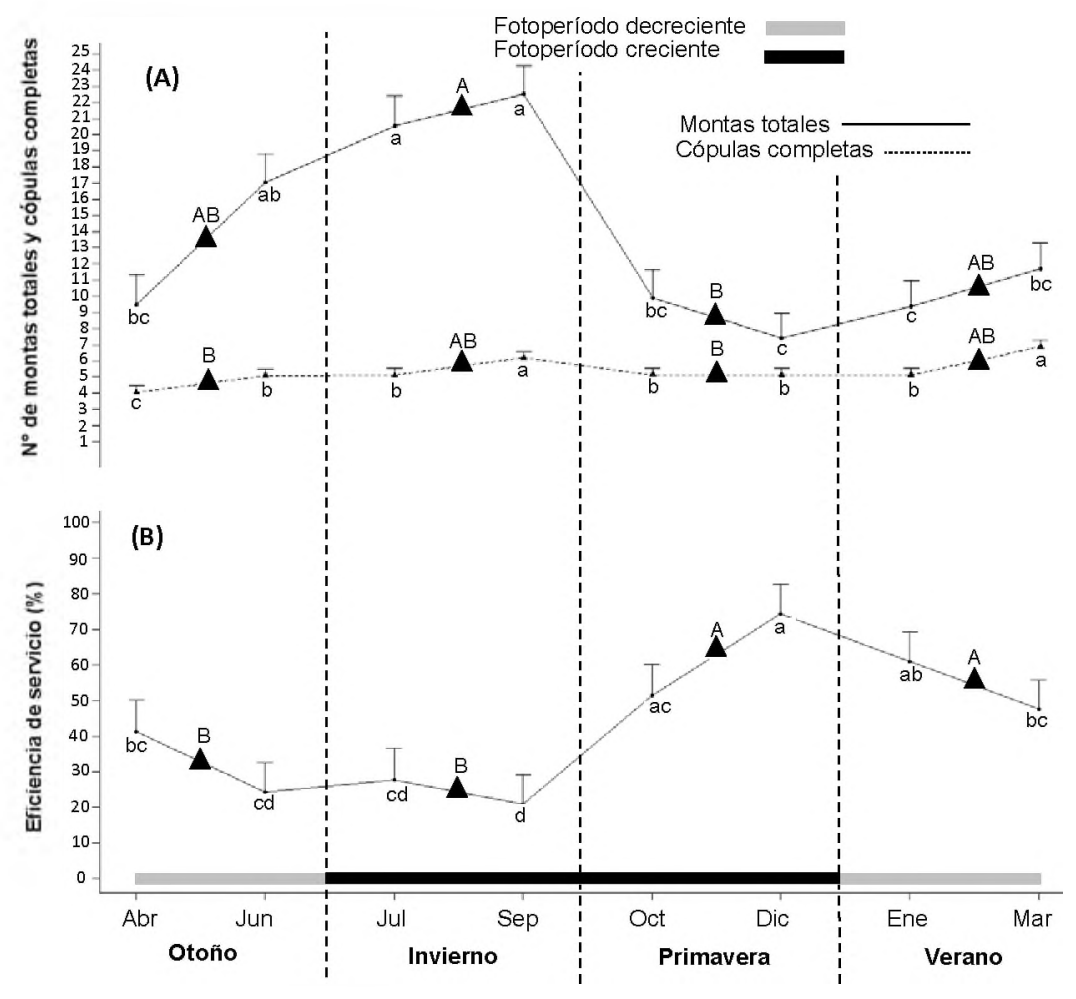

Figura 2. Variación mensual (promedio $\pm \mathrm{EE}$ ) del $\mathrm{N}^{\circ}$ de montas totales, cópulas completas (A) y eficiencia de servicio (\%) (B) ( $\boldsymbol{\Delta}$ contrastes entre estaciones) en carneros Texel. Letras diferentes indican diferencia significativa $(\mathrm{p}<0,05)$.

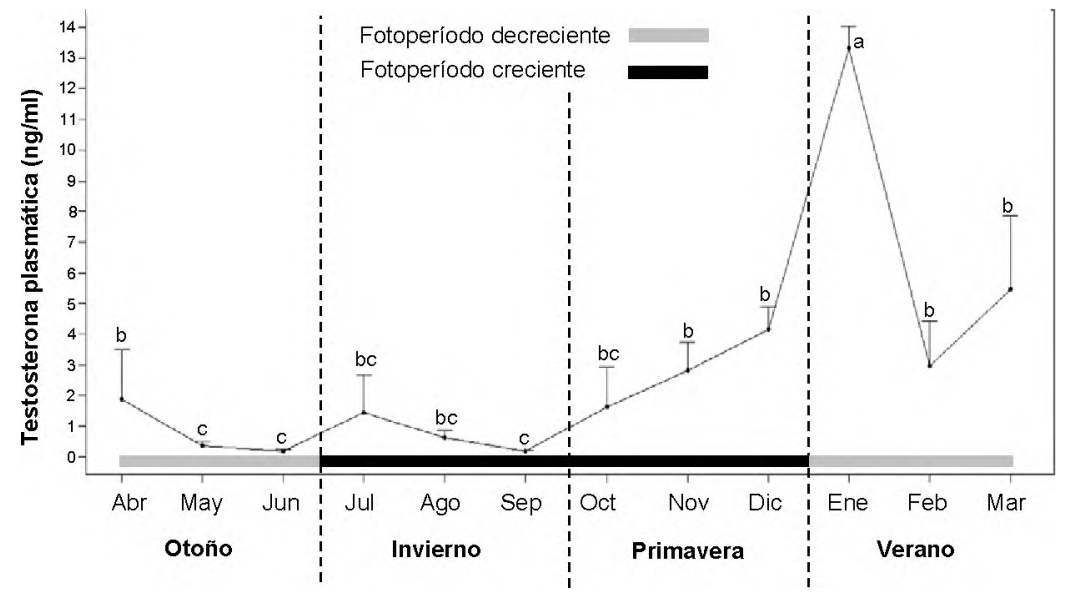

Figura 3. Variación mensual (promedio $\pm \mathrm{EE}$ ) de la concentración de testosterona plasmática en carneros Texel. Letras diferentes indican diferencias significativas $(p<0,05)$. 
Tabla 1. Promedio ( $\pm \mathrm{EE})$ de parámetros seminales de los eyaculados de carneros Texel

\begin{tabular}{lccccccccc}
\hline $\begin{array}{l}\text { parámetros } \\
\text { seminales }\end{array}$ & $\begin{array}{c}\text { VOL } \\
(\mathrm{ml})\end{array}$ & $\mathrm{pH}$ & $\begin{array}{c}\text { CONC } \\
\left(\mathrm{x} 10^{6} \mathrm{ESPZ} / \mathrm{ml}\right)\end{array}$ & $\begin{array}{c}\text { TESPZ } \\
\left(\mathrm{x} 10^{6} \mathrm{ESPZ} / \mathrm{ml}\right)\end{array}$ & $\begin{array}{c}\mathrm{MMM} \\
(0-5)\end{array}$ & $\begin{array}{c}\text { MT } \\
(\%)\end{array}$ & $\begin{array}{c}\text { MIP } \\
(\%)\end{array}$ & $\begin{array}{c}\text { vigor } \\
(0-5)\end{array}$ & $\begin{array}{c}\text { EV } \\
(\%)\end{array}$ \\
\hline Otoño & 1,0 & 7,0 & 3346 & 3196 & 4,4 & 81,8 & 84,3 & 4,0 & 71,0 \\
(n=48) & $\pm 0,1 \mathrm{a}$ & $\pm 0,1$ & \pm 183 & $\pm 245 \mathrm{a}$ & $\pm 0,1 \mathrm{ab}$ & $\pm 2,1 \mathrm{a}$ & $\pm 2,0 \mathrm{a}$ & $\pm 0,2$ & $\pm 1,7 \mathrm{a}$ \\
Invierno & 0,8 & 7,2 & 3039 & 2397 & 4,5 & 77,7 & 84,5 & 4,2 & 73,0 \\
(n=48) & $\pm 0,1 \mathrm{~b}$ & $\pm 0,1$ & \pm 178 & $\pm 232 \mathrm{~b}$ & $\pm 0,2 \mathrm{a}$ & $\pm 2,1 \mathrm{ab}$ & $\pm 2,0 \mathrm{a}$ & $\pm 0,2$ & $\pm 1,7 \mathrm{a}$ \\
Primavera & 0,7 & 7,0 & 3076 & 2323 & 3,9 & 75,2 & 78,0 & 3,9 & 65,1 \\
(n=48) & $\pm 0,1 \mathrm{~b}$ & $\pm 0,0$ & \pm 178 & $\pm 232 \mathrm{~b}$ & $\pm 0,2 \mathrm{~b}$ & $\pm 2,1 \mathrm{~b}$ & $\pm 2,0 \mathrm{~b}$ & $\pm 0,2$ & $\pm 1,7 \mathrm{~b}$ \\
Verano & 0,9 & 6,9 & 3118 & 2949 & 4,3 & 73,1 & 83,7 & 3,9 & 71,2 \\
(n=48) & $\pm 0,1 \mathrm{ab}$ & $\pm 0,0$ & \pm 182 & $\pm 243 \mathrm{ab}$ & $\pm 0,2 \mathrm{ab}$ & $\pm 2,2 \mathrm{~b}$ & $\pm 2,0 \mathrm{a}$ & $\pm 0,2$ & $\pm 1,7 \mathrm{a}$ \\
\hline
\end{tabular}

ab Valores con letras diferentes en la misma columna difieren significativamente (prueba de Tukey; $\mathrm{p}<0,05$ ). VOL: volumen seminal; CONC: concentración espermática; TESPZ: total de espermatozoides; MMM: motilidad de masa microscópica MT: motilidad total; MIP: motilidad individual progresiva; EV: espermatozoides vivos; $\mathrm{pH}$ : $\mathrm{pH}$ seminal.

Tabla 2. Promedio ( \pm EE) de anormalidades espermáticas en semen fresco de carneros Texel.

\begin{tabular}{lcccc}
\hline $\begin{array}{l}\text { parámetros } \\
\text { seminales }\end{array}$ & $\mathrm{EN}(\%)$ & $\mathrm{EACA}(\%)$ & $\mathrm{EAPI}(\%)$ & $\mathrm{EAC}(\%)$ \\
\hline $\begin{array}{l}\text { Otoño } \\
(\mathrm{n}=48)\end{array}$ & $69,1 \pm 3,6$ & $3,0 \pm 0,4 \mathrm{~b}$ & $2,3 \pm 1,6 \mathrm{a}$ & $23,6 \pm 2,6 \mathrm{a}$ \\
$\begin{array}{l}\text { Invierno } \\
(\mathrm{n}=48)\end{array}$ & $76,1 \pm 2,4$ & $1,6 \pm 0,3 \mathrm{~b}$ & $0,4 \pm 0,1 \mathrm{~b}$ & $15,8 \pm 1,4 \mathrm{~b}$ \\
$\begin{array}{l}\text { Primavera } \\
(\mathrm{n}=48)\end{array}$ & $71,8 \pm 2,3$ & $10,5 \pm 0,8 \mathrm{a}$ & $0,1 \pm 0,1 \mathrm{~d}$ & $15,4 \pm 1,5 \mathrm{~b}$ \\
$\begin{array}{l}\text { Verano } \\
(\mathrm{n}=48)\end{array}$ & $76,8 \pm 2,0$ & $11,4 \pm 0,6 \mathrm{a}$ & $0,2 \pm 0,1 \mathrm{c}$ & $8,0 \pm 0,6 \mathrm{c}$ \\
\hline
\end{tabular}

abcd: valores con letras diferentes en la misma columna difieren significativamente (prueba de Tukey; $p<0,05$ ). EN: espermatozoides normales; EACA: espermatozoides con anormalidades a nivel de la cabeza; EAPI: espermatozoides con anormalidades a nivel de la pieza intermedia; EAC: espermatozoides con anormalidades a nivel de la cola.

\section{DISCUSIÓN}

El objetivo del presente estudio fue evaluar las características reproductivas de carneros Texel de dos años de edad, durante un año calendario. A su vez, se propuso conocer la concentración de glucosa y de varias enzimas presentes en el plasma seminal que tuvieran relación con los parámetros de calidad espermática.

El PV aumentó continuamente desde el inicio del estudio (otoño) y alcanzó al finalizar el mismo, el $65 \%$ del peso adulto estimado para la raza. Varios autores demostraron que la medida de la CE presenta una alta correlación con el PT, el VT y la capacidad espermatogénica ${ }^{3}$.

En los carneros Texel, los mayores valores de CE, PT y VT se registraron en el verano y el otoño (fotoperíodo decreciente) y los menores valores, en el invierno y la primavera (fotoperíodo creciente). Este efecto sería la consecuencia de que el inicio del fotoperíodo decreciente está precedido por una estimulación del eje hipotálamo-hipofisario, que conduce a un incremento en la secreción pulsátil de LH y liberación de FSH ${ }^{19}$.

$\mathrm{Al}$ mismo tiempo, aumenta la cantidad de receptores para estas hormonas a nivel testicular y -en con- secuencia- se produce un crecimiento nuclear de las células de Sertoli y multiplicación de las células de Leydig ${ }^{8}$. Otros autores observaron variaciones estacionales similares en razas Texel, Suffolk e Ile-de-France ${ }^{17}$, Suffolk ${ }^{18}$ y Corriedale ${ }^{20}$.

La prueba de CS es útil para detectar individuos con baja líbido o habilidad copulatoria y presenta una moderada heredabilidad $(0,22)$ y alta repetibilidad $(0,72)$ en pruebas sucesivas ${ }^{23}$. En el presente estudio, la CS se manifestó durante todo el año, observándose que el número de cópulas completas no fue diferente entre las estaciones; sin embargo, el número de montas totales fue mayor en el invierno (fotoperíodo creciente), por lo tanto, la eficiencia de servicio fue baja en el otoño e invierno.

Este efecto podría atribuirse a la falta de experiencia sexual al inicio del experimento, por lo que los carneros debieron realizar un mayor número de montas por cada cópula completa. En un grupo de carneros sexualmente inexpertos, se observó que entre el 20 y $35 \%$ son sexualmente inactivos cuando fueron expuestos por primera vez a las hembras ${ }^{21}$. Los resultados obtenidos en este trabajo indicaron que, en carneros jóvenes, una exposición a ovejas sostenida en el tiempo, mejoró su desempeño sexual.

Las máximas concentraciones de testosterona plasmática se determinaron en verano (enero, fotoperíodo decreciente), debido a que en la estación previa a la época reproductiva existe un aumento de receptores de LH a nivel testicular, encargada de estimular la producción de testosterona por parte de las células de Leydig ${ }^{19}$. Concentraciones similares fueron observadas en la raza Pelibuey ${ }^{10} \mathrm{y}$ en las razas Corriedale y Pampinta ${ }^{1}$. Este último estudio fue realizado en la misma latitud que el presente trabajo.

La evaluación del semen fresco permite excluir los eyaculados que no son aptos para las técnicas de reproducción asistida. Los resultados del presente estudio muestran que los carneros Texel de dos años de edad en crecimiento, son capaces de producir semen viable en todas las estaciones, especialmente invierno.

El mayor volumen seminal se registró en el otoño, sin embargo, la concentración espermática no varió entre estaciones, lo cual llevó a obtener un mayor nú- 
Tabla 3. Promedio $( \pm E E)$ de la concentración de glucosa y enzimas en el plasma seminal de carneros Texel.

\begin{tabular}{lcccccc}
\hline $\begin{array}{l}\text { parámetros } \\
\text { enzimáticos }\end{array}$ & $\begin{array}{c}\text { glucosa } \\
(\mathrm{mg} / \mathrm{dl})\end{array}$ & $\begin{array}{c}\text { AST } \\
(\mathrm{U} / \mathrm{I})\end{array}$ & $\begin{array}{c}\text { ALT } \\
(\mathrm{U} / \mathrm{I})\end{array}$ & $\begin{array}{c}\text { GGT } \\
(\mathrm{U} / \mathrm{I})\end{array}$ & $\begin{array}{c}\text { ALP } \\
(\mathrm{U} / \mathrm{I})\end{array}$ & $\begin{array}{c}\mathrm{LDH} \\
(\mathrm{U} / \mathrm{I})\end{array}$ \\
\hline Otoño & 1,3 & 6,9 & 168,8 & 1696 & 68240 & 7542 \\
$(\mathrm{n}=12)$ & $\pm 0,1 \mathrm{~b}$ & $\pm 0,6 \mathrm{a}$ & $\pm 18,2 \mathrm{a}$ & $\pm 77 \mathrm{a}$ & $\pm 5385 \mathrm{ab}$ & $\pm 145 \mathrm{a}$ \\
Invierno & 2,1 & 9,1 & 97,1 & 1644 & 78384 & 6029 \\
$(\mathrm{n}=12)$ & $\pm 0,2 \mathrm{a}$ & $\pm 1,5 \mathrm{a}$ & $\pm 13,3 \mathrm{~b}$ & $\pm 77 \mathrm{a}$ & $\pm 5385 \mathrm{ab}$ & $\pm 123 \mathrm{~b}$ \\
Primavera & 1,8 & 8,4 & 98,1 & 1637 & 82277 & 3293 \\
$(\mathrm{n}=12)$ & $\pm 0,1 \mathrm{a}$ & $\pm 1,0 \mathrm{a}$ & $\pm 13,7 \mathrm{~b}$ & $\pm 76 \mathrm{a}$ & $\pm 6751 \mathrm{a}$ & $\pm 355 \mathrm{~b}$ \\
Verano & 2,4 & 4,7 & 161,4 & 1256 & 59393 & 5561 \\
$(\mathrm{n}=12)$ & $\pm 0,4 \mathrm{a}$ & $\pm 0,5 \mathrm{~b}$ & $\pm 24,6 \mathrm{a}$ & $\pm 72 \mathrm{~b}$ & $\pm 5156 \mathrm{~b}$ & $\pm 561 \mathrm{~b}$ \\
\hline
\end{tabular}

ab Valores con letras diferentes en la misma columna difieren significativamente (prueba de Tukey; $p<0,05$ ). AST:aspartato-aminotransferasa; ALT: alaninaaminotransferasa; GGT: gamma-glutamil-transpeptidasa; ALP: fosfatasa alcalina; LDH: lactato-deshidrogenasa.

mero de espermatozoides totales en esa estación. Similares resultados se registraron en carneros de razas Karakul ${ }^{12}$, Chios y Friesan ${ }^{13}$

Los parámetros cinéticos espermáticos disminuyeron principalmente en la primavera, producto del fotoperíodo creciente y no se observó una relación entre dichos parámetros y las anormalidades a nivel de la cola, coincidiendo con lo informado por Colas y Guerin en $1980^{5}$.

Los porcentajes totales de anormalidades espermáticas no variaron entre estaciones; sin embargo, el mayor porcentaje de anormalidades a nivel de la cabeza se encontró en el verano, debido probablemente al estrés térmico que afectó la termorregulación testicular ${ }^{16}$. En resumen, los mejores valores observados en las características seminales fueron en el otoño e invierno.

La composición de azúcares del plasma seminal es fundamental para el metabolismo y la motilidad de los espermatozoides. Luego de la eyaculación, los azúcares son consumidos por los espermatozoides, por lo tanto, existe una correlación negativa entre la concentración de glucosa y el total de espermatozoides.

En el presente estudio se observó una menor concentración de glucosa en el otoño, coincidentemente con el mayor número de espermatozoides totales y el mayor porcentaje de motilidad total. Esta relación podría interpretarse considerando que, a mayor número de espermatozoides motiles, aumenta el consumo de glucosa ${ }^{29}$.

La actividad de las enzimas transaminasas AST y ALT se relaciona negativamente con la motilidad, la concentración espermática y la estabilidad de la membrana plasmática del espermatozoide ${ }^{7}$. Estas relaciones no se observaron durante este experimento y teniendo en cuenta que son enzimas de origen testicular y epididimario, probablemente exista una falta de homogeneidad en el metabolismo de la AST y ALT en los carneros jóvenes.

Por otro lado, fue observado que los incrementos de AST y ALT se asociaron con el incremento de las alteraciones o anormalidades de los espermatozoides ${ }^{25}$. En el presente trabajo se observó una relación positiva entre la concentración de ALT y las anormalidades espermáticas de la pieza intermedia y la cola en el otoño

La enzima GGT no es necesaria per se para la función reproductiva, pero cumple una función importante en el sistema de glutatión peroxidasa, que está implicada en la protección de los espermatozoides contra los radicales libres ${ }^{30}$. La baja concentración de glutatión peroxidasa por falta de GGT produce espermatozoides defectuosos y disminución en la motilidad espermática ${ }^{9}$.

La baja concentración de GGT determinada en el verano coincidió con el mayor porcentaje de anormalidades espermáticas a nivel de la cabeza y con el menor porcentaje de motilidad total en la misma estación. En consecuencia, la determinación de la concentración de GGT en el plasma seminal, podría utilizarse como un marcador de funcionalidad prostática e indicador de la presencia de anormalidades espermáticas; sin embargo, se requieren concentraciones elevadas de GGT en el plasma seminal para mitigar el efecto de los radicales libres.

Las enzimas ALP y LDH están involucradas en las reacciones glucolíticas, en las cuales proveen energía en forma indirecta para la sobrevivencia, la motilidad y la fertilidad de los espermatozoides ${ }^{28}$. En este estudio, las mayores concentraciones de ALP en el otoño, invierno y primavera coincidieron con los mayores valores de motilidad individual progresiva, lo cual indicaría que la enzima ALP tiene una relación positiva con la cinética de los espermatozoides. Sin embargo, otros autores determinaron una correlación negativa entre los niveles de ALP y la motilidad espermática en búfalos (Bubalus bubalis) ${ }^{6}$

En el equino, se observó que la concentración de ALP presentó una correlación positiva con la concentración espermática, por lo cual se postuló que los testículos y el epidídimo son los responsables de la producción de ALP ${ }^{14}$. Sin embargo, esta relación no se observó en el presente estudio, probablemente porque la ALP en el carnero proviene principalmente de las vesículas seminales y en menor medida de los testículos y epidídimos ${ }^{2}$

La enzima LDH se localiza a nivel del citosol y en las mitocondrias espermáticas. Al ser una enzima intracelular, el incremento de la concentración en plasma seminal indicaría anormalidades o daño en la membrana espermática ${ }^{24}$. En el presente estudio se observó una relación entre la concentración de LDH y los mayores porcentajes de anormalidades espermáticas a nivel de la pieza intermedia y de la cola en el otoño.

En consecuencia, la determinación de la concentración de LDH en el plasma seminal podría utilizarse como un indicador indirecto de la presencia de anormalidades espermáticas. Por otro lado, una correlación 
negativa entre el nivel de LDH y el porcentaje de espermatozoides vivos fue observada en carneros de raza Moghani ${ }^{31}$.

Esta relación no se observó en este experimento, ya que la mayor concentración de LDH se registró en el otoño en coincidencia con un elevado porcentaje de espermatozoides viables. El incremento de los niveles de LDH en el otoño, probablemente no se deba a la mortalidad espermática en el eyaculado, sino que podría ser atribuido a un signo de degeneración y destrucción a nivel de las espermatogonias ${ }^{4}$, indicando algún grado de inmadurez en la espermatogénesis cuando los carneros no alcanzaron la madurez sexual.

En conclusión, el estudio demuestra que las características reproductivas evaluadas en carneros Texel de dos años de edad, presentan variaciones estacionales marcadas y soportan la hipótesis que el otoño e invierno son las estaciones del año con la mejor aptitud reproductiva en la latitud estudiada. Además, algunas enzimas del plasma seminal podrían ser de utilidad para evaluar la calidad seminal.

Agradecimientos. A1 personal auxiliar del grupo de reproducción animal de la EEA-INTA Balcarce por el manejo y cuidado de los animales. Este trabajo fue financiado por INTA (Proyecto PNSA 1115053) y Fundación ArgenINTA.

\section{REFERENCIAS}

1. Aller JF, Aguilar D, Vera T, Almeida GP, Alberio RH. 2012. Seasonal variation in sexual behavior, plasma testosterone and semen characteristics of argentine pampinta and corriedale rams. Spanish J Agr Res 10: 345-352.

2. Asadpour R. 2012. Relationship between mineral composition of seminal plasma and semen quality in various ram breeds. Acta Sci Vet 40: 1027.

3. Bailey TL et al. 1998. Caliper and ultrasonographic measurements of bovine testicles and a mathematical formula for determining testicular volume and weight in vivo. Theriogenology 49: 581-594.

4. Burgos C et al. 1995. Intracellular localization of the testicular and sperm-specific lactate dehydrogenase isozyme C4 in mice. Biol Reprod 53: 84-92.

5. Colas G, Guerin, Y. 1980. Variations saisonnières de la qualité du sperme chez le bélier Ile-de-France. I. Etude de la morphologie cellulaire et de la motilité massale. Reprod Nutr Dev 20: 1789-1799

6. Dhami AJ, Kodagali SB. 1990. Freezability, enzyme leakage and fertility of buffalo spermatozoa in relation to the quality of semen ejaculates and extenders. Theriogenology 34: 853-863.

7. Flipse RJ. 1960. Metabolism of bovine semen. IX: Glutamic-oxaloacetic and glutamic-pyruvic transaminase activities. J Dairy Sci 43: 773-776.

8. Garnier DH et al. 1981. LH, prolactin, testosterone, testis weights and Leydig cells in the adult normal ram during breeding season. Int J Androl Suppl 3: 44-45.
9. Gharagozloo P, Aitken RJ. 2011. The role of sperm oxidative stress in male infertility and the significance of oral antioxidant therapy. Hum Reprod 26: 1628-1640.

10. González A, Foote WC, Murphy BD, Ortega E. 1992. Seasonal variations in circulating testosterone and luteinizing hormone in Pelibuey lambs. Small Rum Res 8: 233242

11. Ibarra D, Laborde D, Olivera J, Vanlier E, Burgueño J. 1999. Comparación de tres pruebas para medir la capacidad de servicio en carneros adultos. Arch Med Vet 31: 189-196.

12. Kafi M, Safdarian M, Hashemi M. 2004. Seasonal variation in semen characteristics, scrotal circumference and libido of persian karakul rams. Small Rum Res 53: 133-139.

13. Karagiannidis A, Varsakeli S, Alexopoulos C, Amarantidis I. 2000. Seasonal variation in semen characteristics of Chios and Friesian rams in Greece. Small Rum Res 37: $125-130$

14. Kareskoski M, Katila T. 2008. Components of stallion seminal plasma and the effects of seminal plasma on sperm longevity. Anim Reprod Sci 107: 249-256.

15. Khokhar BS, Singh M, Chaudhary KC. 1987. Transaminases in cattle and buffalo (Bubalus bubalis) semen in relation to fertility and seminal characteristics during moderate and colder seasons. Anim Reprod Sci 13: 177-182.

16. Lue YH et al. 1999. Single exposu re to heat induces stagespecific germ cell apoptosis in rats: role of intratesticular testosterone on stage specificity. Endocrinology 140: 1709-1717.

17. Mandiki SN, Derycke G, Bister JL, Paquay R. 1998. Influence of season and age on sexual maturation parameters of Texel, Suffolk and Ile-de-France rams: testicular size, semen quality and reproductive capacity. Small Rum Res 28: 67-79.

18. Milczewski V, Chahad ES, Spercoski KM, Morais RN, Soccol VT. 2015. Quantifying the effect of seasonality on testicular function of Suffolk ram in lower latitude. Small Rum Res 124: 68-75.

19. Pelletier J, Garnier DH, Reviers MM, Terqui M, Ortavant R. 1982 Seasonal variation in $\mathrm{LH}$ and testosterone release in rams of two breeds. $J$ Reprod Fertil 64: 341-346.

20. Pérez CR et al. 1997. Reproductive seasonality of Corriedale rams under extensive rearing conditions. Acta Vet Scand 38: 109-117.

21. Price EO, Estep DQ, Wallach SJ, Dally MR. 1991. Sexual performance of rams as determined by maturation and sexual experience. J Anim Sci 69: 1047-1052.

22. R-Core Team. 2016. A language and environment for statistical computing, Foundation for Statistical Computing, Vienna, Austria. https://www.R-project.org.

23. Snowder GD, Stellflug JN, Vanvleck LD. 2002. Heritability and repeatability of sexual performance scores of rams. J Anim Sci 80: 1508-1511.

24. Stallcup OT, Roussel JD. 1965. Development of the lactic dehydrogenase enzyme system in the testes and epididymis of young dairy bulls. J Dairy Sci 48: 1511-1516.

25. Taha TA, Abdel EI, Ayoub MA. 2000. Monthly variations in some reproductive parameters of Barki and Awassi rams throughout 1 year under subtropical conditions. 
Biochemical properties of seminal plasma. Anim $S_{c i} 71$ : 325-332.

26. Töpfer PE, Ekhlasi HM, Kirchhoff C, Leeb T, Sieme H. 2005. The role of stallion seminal proteins in fertilization. Anim Reprod Sci 89: 159-170.

27. Troedsson MH et al. 2005. Components in seminal plasma regulating sperm transport and elimination. Anim Reprod Sci 89: 171-186.

28. Turner RM, McDonnell SM. 2003. Alkaline phosphatase in stallion semen: characterization and clinical applications. Theriogenology 60: 1-10.
29. Williams AC, Ford WC. 2001. The role of glucose in supporting motility and capacitation in human spermatozoa. $J$ Androl 22: 680-695.

30. Zalata A, Hafez T, Mahmoud A, Comhaire F. 1995. Relationship between resazurin reduction test, reactive oxygen species generation, and $\gamma$-glutamyl-transferase. Hum Reprod 10: 1136-1140.

31. Zamiri MJ, Khalili B, Jafaroghli M, Farshad A. 2010. Seasonal variation in seminal parameters, testicular size, and plasma testosterone concentration in iranian Moghani rams. Small Rum Res 94: 132-136. 